\title{
Cigarette Smoke Induces Epidermal Growth Factor Receptor-Dependent Redistribution of Apical MUC1 and Junctional $\beta$-Catenin in Polarized Human Airway Epithelial Cells
}

Ying-Ting Chen, * Marianne Gallup, * Karina Nikulina, ${ }^{*}$ Stanislav Lazarev, ${ }^{*}$ Lorna Zlock, ${ }^{\dagger}$ Walter Finkbeiner, ${ }^{\dagger}$ and Nancy McNamara*‡

From the Francis I. Proctor Foundation," and the Departments of Pathology, and Anatomy and Ophthalmology, University of California, San Francisco, California

Cigarette smoke (CS) accounts for nearly $90 \%$ of lung cancer deaths worldwide; however, an incomplete understanding of how CS initiates preneoplastic changes in the normal airway hinders early diagnosis. Short-term exposure to CS causes aberrant activation of epidermal growth factor receptor (EGFR) and canonical Wnt/ $\beta$-catenin signaling pathways in human bronchial epithelial (HBE) cells. We hypothesize that this response is elicited through the disruption of spatially segregated cell membrane proteins in the polarized airway epithelium. Using an in vitro model of highly differentiated HBE cells, we observed membrane characteristics consistent with the native airway, including the presence of a membrane mucin, MUC1, at the apical cell pole, $\beta$-catenin at the apicallateral membrane, and EGFR at the basolateral membrane. Following exposure to smoke, intercellular spaces enlarge and cilia disappear. This histopathology is accompanied by molecular events that include perinuclear trafficking of basolateral EGFR, EGFR phosphorylation, pEGFR-mediated phosphorylation of MUC1's cytoplasmic tail (CT), loss of E-cadherin/ $\beta$ catenin complexes at the adherens junctions (AJs), intracellular formation and nuclear shuffling of $\beta$-catenin/MUC1-CT complexes, and, ultimately, upregulation and nuclear localization of Wnt nuclear effector, Lef-1. In the presence of EGFR inhibitor, AG1478, CS-induced histopathology and molecular events were inhibited. These data point to EGFR as a portal through which CS mediates its damaging effects on AJ-mediated cell polarity and activation of canonical Wnt/ $\beta$-catenin signaling. (Am J Pathol 2010, 177:1255-1264; DOI: 10.2353/ajpath.2010.091129)
Approximately 5.5 trillion cigarettes produced globally each year are smoked by more than 1.1 billion people; one-sixth of the world population. ${ }^{1}$ In the United States alone, cigarette smoke (CS) is linked to nearly 500,000 deaths per year, and approximately half of all regular smokers die of smoking-related complications. ${ }^{2}$ As the major single cause of cancer mortality, CS accounts for $87 \%$ of lung cancer and causes $\sim 30 \%$ of all cancerrelated deaths in the United States. ${ }^{3,4}$ While there is little debate surrounding the negative impact of CS on public health, the mechanisms whereby noxious chemicals in CS perturb the normal airway epithelium and lead to airway remodeling, chronic inflammation, and malignant transformation are not clearly defined.

The human bronchial epithelium (HBE), is a pseudostratified, mucosal epithelium, comprising ciliated columnar cells, mucus-producing goblet cells, and basal progenitor cells. $^{5}$ The HBE plays an important physiological role in innate immunity by providing a physical barrier to environmental insults and, if breached, initiating a defense response via an array of receptor-mediated events. ${ }^{6}$ Barrier function of the HBE is maintained through the cooperation of two mutually exclusive structural units: trans-membrane mucins on the apical cell surface of ciliated cells (mucociliary barrier $)^{7}$ and subapical junctional complexes consisting of tight junctions (TJS) and adherens junctions (AJs) on the lateral membranes. ${ }^{8}$ The unique apicolateral localization of these junctional complexes mediates cell-cell adhesion and sterically polarizes HBE cells into apical and basolateral plasma membrane domains, on which transmembrane mu-

Supported by American Cancer Society grant 115502-RSG-08-13601-CNE.

This manuscript is written in memory of Dr. Carol B. Basbaum, whose outstanding mentorship in mucin biology and personal battle against cancer inspired this work.

Accepted for publication May 25, 2010.

Address reprint requests to Nancy McNamara, O.D., Ph.D., University of California, San Francisco, Francis I. Proctor Foundation, Room S334, 513 Parnassus Avenue, Box 0412, San Francisco, CA 94143. E-mail: nancy. mcnamara@ucsf.edu. 
cin, MUC1, and epidermal growth factor receptor (EGFR) serve as landmarks, respectively. This polarized cytoarchitecture is essential for preserving mucosal integrity and initiating receptor-mediated events in response to a variety of extrinsic signals. Among cell surface receptors most commonly expressed by HBE cells, the EGFR family serves as a principal regulator of tissue repair in response to stress or injury. ${ }^{6}$

Recent evidence suggests that structural components of AJs and transmembrane mucins have dual physiological functions. In addition to supporting the epithelial architecture, some structural components dynamically participate in signal transduction through context-dependent interactions with a variety of signaling partners. In a homeostatic epithelium, $\beta$-catenin cooperates with E-cadherin to form AJs and maintain cell polarity. ${ }^{9}$ In airway regeneration and oncogenic transformation, membranous $\beta$-catenin translocates to the cell nucleus where it interacts with the transcription factor family T-cell factor (TCF)/lymphoid enhancer factor (LEF) to activate the canonical Wnt signaling pathway. ${ }^{10,11}$ Similar to $\beta$-catenin, the cytoplasmic tail (CT) of transmembrane mucin, MUC1, supports the structural barrier during homeostasis but trans-locates from the apical membrane in response to a variety of insults to generate intracellular instructions. ${ }^{12,13} \mathrm{~A}$ prerequisite for signal transduction through these two structural molecules is intracellular translocation from the polarized plasma membrane.

CS exposure has been shown to cause a wide spectrum of preneoplastic histological airway lesions, ranging from hyperplasia, metaplasia, dysplasia, to carcinoma in situ. ${ }^{14} \mathrm{~A}$ plethora of molecular abnormalities has been detected in both histologically healthy and preneoplastic respiratory epithelia of smokers. ${ }^{14}$ The theory that CS causes a diffuse nonneoplastic field of molecular injury throughout the entire respiratory tract in the preneoplastic stage of multistep carcinogenesis is referred as "field cancerization." 15-17 Intriguingly, redistribution of EGFR, mucins, and $\beta$-catenin are among the most common "altered" molecular features observed in the normal-appearing preneoplastic airway epithelium of "healthy" smokers. ${ }^{18-20}$ Moreover, in company with the redistribution of EGFR and $\beta$-catenin, short-term exposure of HBE cell monolayers to CS has been shown to initiate signaling events that include aberrant activation of both EGFR and canonical Wnt/ $\beta$-catenin signaling. ${ }^{21-23}$ While EGFR and Wnt are known mediators of CS-induced airway diseases, the specific events that coordinate cytoarchitectural damage and aberrant activation of protumor signaling are unclear.

To identify the onset of preneoplastic changes in response to CS, a detailed knowledge of the early events leading to histological alteration and aberrant activation of protumor signaling pathways is necessary. Given the frequent molecular alteration of EGFR, mucins, and $\beta$-catenin in preneoplastic lesions, we hypothesized that the structural role of these membrane proteins is disrupted by CS, and that their redistribution to the intracellular compartment provides a driving force that activates pro-tumor signaling. Using a three-dimensional (3D) in vitro model of the airway epithelium, we demonstrated that CS induces an EGFR-dependent cytoarchitectural disorganization that leads to the translocalization of polarity landmark proteins, apical MUC1 glycoprotein and junctional $\beta$-catenin, from the cell membrane to the cytoplasm and nucleus. Intranuclear accumulation of $\beta$-catenin subsequently leads to aberrant activation of canonical Wnt/ $\beta$-catenin signaling. The potential link between cell polarity and aberrant activation of intracellular signaling in response to CS is discussed.

\section{Materials and Methods}

\section{Culture of HBE Cells}

Human tissue was handled according the Declaration of Helsinki and was approved by the University of California Committee for Human Research. Culture of primary HBE cells was established using methods previously described. ${ }^{24}$ Briefly, tracheal tissue strips from human donors were rinsed three times in PBS containing $5 \mathrm{mmol} / \mathrm{L}$ DTT and twice in PBS. The bronchial epithelium was separated from the underlying stroma using enzymatic digestion followed by vigorous agitation to dislodge the epithelial sheets. Single cells were isolated from epithelial sheets after a short incubation in $0.25 \%$ trypsin/EDTA. Primary HBE cells were plated $1 \times 10^{5}$ cells $/ \mathrm{cm}^{2}$ onto Transwell polycarbonate membranes, $0.4-\mu \mathrm{m}$ pore diameter; Corning, Inc., NY, precoated with human placental collagen (15 mg/ $\mathrm{cm}^{2}$; Sigma-Aldrich, St. Louis, MO). Cells were grown in defined ALI medium at an air liquid interface producing highly differentiated and functional replicas of the native airway epithelium for approximately 18 days. ${ }^{24,25}$ Experiments were conducted using primary cultures from 16 different human donors to confirm consistency.

\section{Preparation of CS-Conditioned Medium}

CS-conditioned medium was generated in specially designed animal exposure chambers operated by Dr. Kent Pinkerton at the University of California, Davis. ${ }^{26}$ Cell culture medium was exposed to smoke for six continuous hours in an open Petri dish placed in the exposure chamber. The smoke medium thus obtained was used fresh or kept frozen at $-20^{\circ} \mathrm{C}$ until use. Cell cultures were exposed to CSconditioned medium at various doses and times to define dose- and time-dependent effects as described below.

\section{MTT Cytotoxicity Assay for HBE Cells}

An MTT assay was performed to determine whether or not the doses of CS-conditioned medium had cytotoxic effect on HBE cells. Primary HBE cells were plated at the density of 5000 cells per well in a 96-well plate. Smokefree medium, $25 \%$ or $50 \%$ CS-conditioned medium was used to cultivate cells for 48 and 72 hours with MTT salts at the concentration recommended by the manufacturer (Roche, Branford, CT). Colorimetric analysis was performed in an ELISA plate reader set at $570 \mathrm{~nm}$ to quantify the activity of MTT reducing enzymes. Three independent experiments were conducted using cells obtained from three different donors and five technical replicates per do- 
nor. Data were expressed as mean \pm SD. Data were analyzed using analysis of variance to compare differences in MTT between control and smoke-exposed cells. We adjusted for multiple comparisons using the Bonferroni correction. All analyses were done using the statistical software Stata 9.0 (College Station, TX) for Macintosh.

\section{Cell Fractionation, Immunoblotting, and Immunoprecipitation}

Proteins from cytoplasmic, nuclear, or membrane fractions were extracted using a compartmental protein extraction kit (Millipore, Billerica, MA) according to the manufacturer's instructions. Protein concentrations were determined using a BCA assay kit (Pierce Biotechnology, Rockford, IL). For immunoblotting, equal amounts of protein were separated by SDS-PAGE on BioRad 4 to $20 \%$ Ready Gels, transferred to nitrocellulose membranes, blocked with TBS/ 0.01\% Tween-20/5\% nonfat dry milk (TBST/M). Blots were incubated with primary antibody [ie, anti- $\beta$-catenin (1: 3000, BD Biosciences, San Jose, CA), anti-E-cadherin (BD Biosciences, 1:2500), anti- $\beta$-actin (1:3000, Biovision, Mountain View, CA), or anti-MUC1-CT2 (a gift from Dr. Sandra Gendler, 1:1500)] overnight at $4^{\circ} \mathrm{C}$. After washing 4 times in TBST/M, blots were incubated in horseradish peroxidase-conjugated secondary antibody for 1 hour and visualized using the ECL chemiluminescence detection system (Amersham Life Sciences, Piscataway, $\mathrm{NJ}$ ). For immunoprecipitation, equal amounts of protein were incubated with primary antibody and protein agarose $A / G$ beads overnight at $4^{\circ} \mathrm{C}$. After washing four times, beads were diluted in $20 \mu \mathrm{l} 2 \times$ SDS sample buffer and processed by Western blotting as described above.

\section{Laser Scanning Confocal Microscopy, Immunofluorescence, and Immunohistochemistry}

Cell culture inserts were fixed in $4 \%$ paraformaldehyde and embedded in paraffin using standard techniques. Sections (5 $\mu \mathrm{m})$ were cut with a rotary microtome, stained with hematoxylin-eosin, and photographed with a light microscope equipped with a digital camera. Unstained sections were used for immunofluorescence. Before exposure to antibodies, the inserts were subjected to antigen retrieval by incubation in $10 \mathrm{mmol} / \mathrm{L}$ Tris, $1 \mathrm{mmol} / \mathrm{L}$ EDTA, $0.05 \%$ Tween-20, pH 9.0 for 20 minutes at $85^{\circ} \mathrm{C}$. The slides were permeabilized with $0.2 \%$ Triton-X-100 and blocked with $5 \%$ normal goat serum for 30 minutes. For immunofluorescence, sections were incubated overnight at $4^{\circ} \mathrm{C}$ with primary antibodies (anti- $\beta$-catenin, 1:200, anti-E-cadherin 1:200, anti-MUC1-CT2, 1:500, and anti-Lef-1, 1:200), followed by incubation with the appropriate secondary antibodies conjugated with either Alexa Fluor 488 (Invitrogen, Carlsbad, CA) or Cy3 (Jackson ImmunoResearch, West Grove, PA) for one hour at room temperature with nucleus counterstained with DAPI. For immunohistochemistry, the same procedure for primary antibody (anti-EGFR 1:200) was followed by standard avidin-biotin peroxidase complex staining with diaminobenzidine (DAB) as the chromogen. DAB colorimetric samples were photographed by Nikon Eclipse Ti-E microscope using transmitted light. Immunofluorescent samples were photographed with a Nikon Eclipse Ti-E epifluorescence microscope or Nikon C1Si laser scanning confocal microscope. Intensity of E-cadherin immunofluorescence was analyzed by NIS-Elements BR 2.30 software, using an image analysis tool to quantify fluorescent intensity at 9 AJs adjoining 10 continuous HBE cells in three randomly chosen $20 \times$ power fields for each donor. Analysis of variance was used to identify significant differences between control and smoke-exposed cells.

\section{Transcriptional Profiling of HBE Cells Using TaqMan Gene Expression Assays}

Total RNA was extracted from primary HBE cells with or without smoke exposure for 48 hours, using an RNeasy Mini RNA isolation kit (Qiagen, Piscataway, NJ). Total RNA was eluted from mini columns with $30 \mu \mathrm{l}$ of RNase-free water. The amount of total RNA was quantified by optical density measurements at $260 \mathrm{~nm}\left(\mathrm{OD}_{260}\right)$. Starting from $1 \mu \mathrm{g}$ of total RNA, $80 \mu$ l of cDNA was synthesized by using $\mathrm{RT}^{2}$ First Strand Kit (SuperArray), which contains Oligo d(T $)_{16}$, RNase inhibitor, deoxyNTPs mixture and MultiScribe reverse transcriptase. The reaction was performed for 10 minutes at $25^{\circ} \mathrm{C}, 15$ minutes at $42^{\circ} \mathrm{C}$, and 5 minutes at $95^{\circ} \mathrm{C}$. cDNA was stored at $-20^{\circ} \mathrm{C}$ until use. To compare the relative abundance of LEF1 transcripts expressed, a TaqMan Probe fluorogenic 5'nuclease chemistry-based gene expression assay with exon boundary-crossing primers (assay ID: Hs01547248_m1) was applied. Real-time PCR was performed using thermal cycling conditions at $95^{\circ} \mathrm{C}$ for 10 minutes as the initial step, followed by 40 cycles of 15 seconds at $95^{\circ} \mathrm{C}$ and 1 minute at $60^{\circ} \mathrm{C}$ for amplification in ABI Prism 7300 Real-Time PCR System (Applied Biosystems, Fort City, CA). All assays were performed and compared in four technical replicates to house keeping genes (GAPDH). Data were obtained as threshold thermal cycle (Ct) value. Three biological replicates (three donors) were used for obtaining Ct data in both smoke and control groups. After normalization with a housekeeping gene, the fold change derived from $\Delta \Delta \mathrm{Ct}$ of smoke versus control was examined by analysis of variance with a $P$ value $<0.05$ being considered significant. Positive and negative quality controls for reproducibility, reverse transcription and genomic DNA contamination were assessed and found to be acceptable.

\section{Results}

Cigarette Smoke Induces Epithelial Cell Proliferation and Loss of Polarity in a 3D, Pseudo-Stratified Model of the Human Bronchial Epithelium

To determine whether doses of CS used in the study had cytotoxic effects on HBE cells, we used MTT assays to 
assess both dose- and time-dependent effects of smoke exposure. Compared with untreated controls, cytotoxicity was not detected in CS-treated cells at any dose of smoke $(25 \%$ or $50 \%$ Smk) or at any time point ( 48 or 72 hours) using cells obtained from three donors (Figure 1A). These results indicated that the CS-conditioned medium used in our experimental set-up did not cause nonspecific cell death (eg, apoptosis) that could potentially confound smoke-specific effects on cell morphology or intracellular signaling. In the absence of smoke we observed an intact pseudostratified epithelium with cellular characteristics of differentiation, including large, columnar, ciliated cells with low nucleus-to-cytoplasmic $(\mathrm{N} / \mathrm{C})$ ratio at the apical layer, and smaller basal cells with larger horizontally oriented nuclei (Figure 1B, Con). On exposure to $50 \%$ Smk for 24 hours, cilia on the apical surface of columnar cells largely disappeared and intercellular spaces increased between the basal cells (Figure 1B, 50\% Smk, 24 hours). By 48 hours, apicobasal polarization was replaced by disorganized cell layers containing mainly basal-cell like cells with significant surface cell shedding (Figure 1B, 50\% Smk, 48 hours). By 72 hours, apical cell shedding increased, columnar cells completely disappeared and cell-cell junctions were severely disrupted (Figure 1B, 50\% Smk, 72 hours). Taken together, CS disrupted cell polarity and decreased cell-cell adhesion in the 3D pseudostratified HBE cell culture system.

\section{Loss of Cell Polarity in Response to CS Is Associated with Destabilization of the AJ and Loss of E-Cadherin}

AJs consisting of E-cadherin and $\beta$-catenin are located at the subapical intercellular junction. Because AJs are prerequisite in maintaining a polarized airway epithelium, we investigated the effect of CS on E-cadherin and $\beta$-catenin. Stratified epithelia of primary HBE cells were incubated for 48 and 72 hours, with or without $50 \%$ Smk. Immunoblotting of the membranous fraction from cell lysates revealed a significant decrease in E-cadherin following smoke exposure at both time points (Figure 2A). Immunofluorescence showed a time-dependent decrease in E-cadherin at the AJs in response to CS. Junctional E-cadherin was decreased in approximately half of the epithelial cells by 48 hours and nearly all of the cells by 72 hours (Figure 2B). We further used confocal imaging with a fixed laser exposure time to measure E-cadherin expression in the presence and absence of $50 \%$ Smk ( $n=3$ donors). We provided representative confocal images from a single donor at 48 hours (Figure 2C, upper panels). Using these images we quantified E-cadherin immunofluorescence signal strength at nine AJs adjoining 10 continuous HBE cells. A representative example of signal strength at each $\mathrm{AJ}$ in control (Con) and smoke-exposed (Smk) cells is provided (Figure 2C, lower left panel). Signal intensity of membrane E-cadherin in response to smoke was normalized to control cells at each time point ( 48 hours and 72 hours) and densitometric analysis used to confirm that reduced expression was statistically significant (Figure 2C, lower right panel).
Moreover, we performed immunoprecipitation on the cell membrane-fractionated cell lysates to show that E-cadherin's direct interaction with $\beta$-catenin at AJs decreased in response to smoke in a dose-dependent manner (Figure 2D). Together, these data suggest smoke causes a loss of E-cadherin/ $\beta$-catenin complexes at the AJs, which is essential for maintaining cell polarity.

\section{Destabilization of the AJs in Response to CS Leads to Intracellular Complex Formation Between Dislocated Apical Mucin MUC1 and Junctional $\beta$-Catenin}

Having shown that CS exposure disrupts key components of the AJ, we went on to examine whether or not CS caused the redistribution of structural landmarks in polarized HBE cells. Using double immunostaining, we examined the subcellular localization of apical membranebound mucin, MUC1, and basolateral AJ component, $\beta$-catenin, in pseudostratified HBE cells. In the absence of smoke, cells showed a highly organized pattern of MUC1-CT expression (red) at the apical cell surface that was segregated from $\beta$-catenin (green) on the lateral membranes of both apical columnar cells and basal cells (Figure 3A, Con). Continuous CS exposure for 48 hours disrupted the spatial segregation of MUC1 and $\beta$-catenin, leading to intracellular translocation of both membrane-bound proteins (Figure 3A, Smk). We observed movement of MUC1-CT from the apical surface to a lateral submembrane location where it colocalized with intracellular $\beta$-catenin (Figure 3A, Smk). As highlighted using a figure inset (Figure 3A, lower right, merged image, Smk 48 hours), intracellularly colocalized MUC1-CT/ $\beta$-catenin was found in the perinuclear region of the cytoplasm (yellow signal denoted with arrows), as well as inside the cell nucleus (white signal denoted using an arrowhead). Subcellular redistribution of MUC1-CT and $\beta$-catenin in response to smoke was accompanied by the loss of normal apical-basal orientation of the ciliated columnar-to-basal HBE cells and replaced by irregularly oriented cells of variable size (Figure 3A, Smk). To test the hypothesis that smoke causes a physical interaction between MUC1-CT and $\beta$-catenin, we performed immunoprecipitation assays using cells obtained from three different donors. MUC1-CT immunoprecipitates from the nuclear fraction were immunoblotted with anti- $\beta$-catenin antibody. Consistently, MUC1-CT/ $\beta$-catenin complexes accumulated in the nucleus in response to smoke (Figure $3 \mathrm{~B})$. Normalized to MUC1-CT/ $\beta$-catenin complexes in control immunoprecipitates (considered as onefold), densitometric analysis showed an average 2.3-fold increase in nuclear MUC1-CT/ $\beta$-catenin complexes in CSexposed cells by 48 hours (Figure 3B). Moreover, in an independent experiment we demonstrated that the nuclear accumulation of MUC1-CT/ $\beta$-catenin complex was CS dose-dependent (Figure $3 C$ ). These data indicate that CS causes the intracellular formation and nuclear accumulation of MUC1-CT/ $\beta$-catenin complexes, in company with the loss of cell polarity. 
A

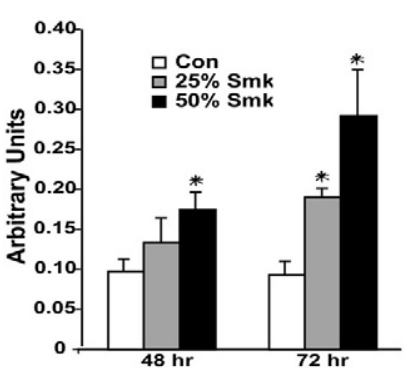

A

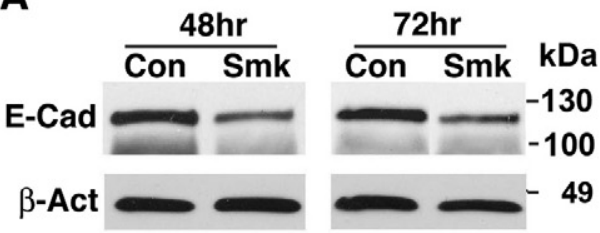

C

Con $48 \mathrm{hr}$
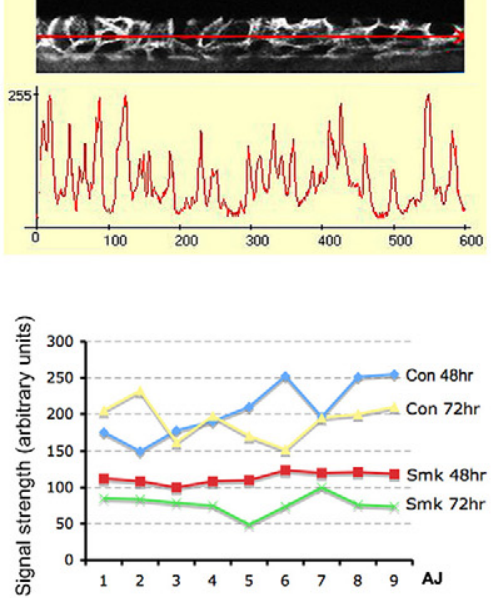

B

Con
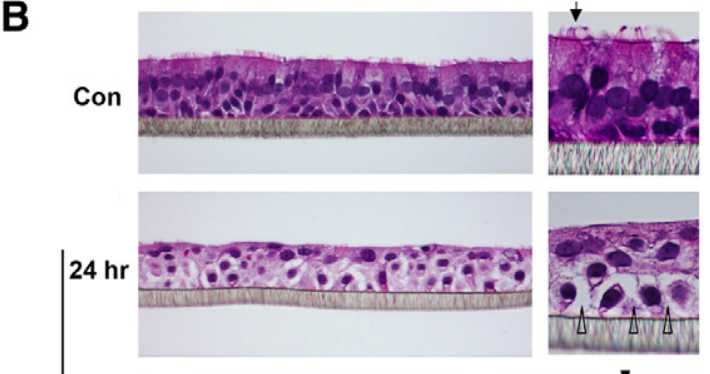

$48 \mathrm{~h}$
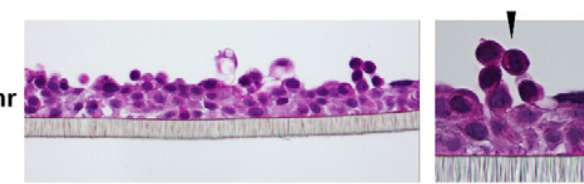

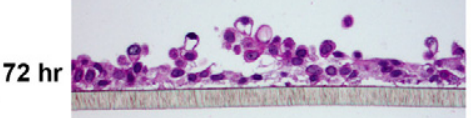

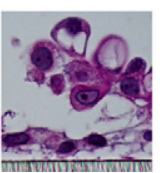

Figure 1. CS causes preneoplastic structural changes in a three-dimensional model of primary, differentiated, pseudostratified HBE cells A: Cytotoxicity was evaluated by MTT assay using HBE cells obtained from three individual donors. Cells were treated with $25 \%$ and 50\% CS-conditioned medium (Smk) or basal control medium (Con) for 48 or 72 hours. Data are expressed as the mean $\pm \mathrm{SD}$ in arbitrary units. Statistical significance was determined using analysis of variance. ${ }^{*} P<0.05$. B: H\&E staining of pseudostratified HBE cells incubated with $50 \%$ CS-conditioned medium (Smk) or basal control medium (Con) for 24, 48, and 72 hours. Black arrow indicates apical cilia in columna cells. Black arrowheads indicate detached epithelial cells. Open arrowheads indicate enlarged intercellular spaces among basal HBE cells. Of note, loss of cell polarity and epithelial damage are time dependent. Apical cilia disappear and intercellular spaces between basal cells increase by 24 hours; apical surface breaks down and cell-cell junctions are disrupted throughout the epithelium by 48 hours; basal cells begin to detach from culture membrane inserts by 72 hours. Scale bar $=50 \mu \mathrm{m}$.
B
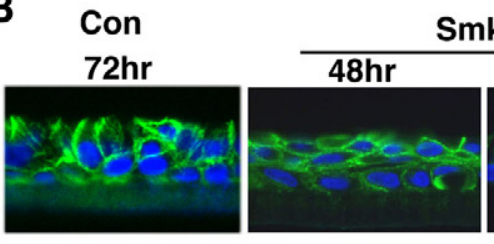

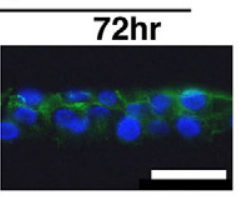

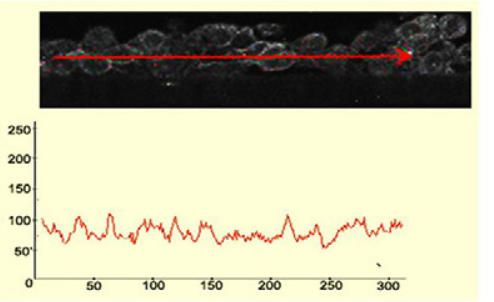

Smk 48hr

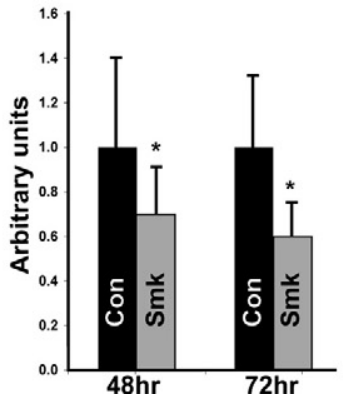

Figure 2. CS disrupts cell polarity by dissociating E-cadherin and $\beta$-catenin at adherens junctions. A: Pseudostratified HBE cells were incubated for 48 and 72 hours with $50 \%$ CS-conditioned medium (Smk) or basal control (Con). Western blot for E-cadherin was performed on membranous fractions of cell lysates. Protein was loaded in equal amounts for all groups, using $\beta$-actin as the loading control. A representative immunoblot from one of three donors is shown. B: Immunofluorescent staining of E-cadherin (green) in polarized HBE cells exposed to 50\% Smk for 48 and 72 hours or untreated controls. Cell nuclei were counterstained with DAPI (blue). Scale bar $=50 \mu \mathrm{m}$. C: Confocal image analysis to quantify immunosignal intensity of membrane E-cadherin in HBE cells exposed to control or $50 \%$ Smk for 48 hours and 72 hours. Signal strength of E-cadherin fluorescence (arbitrary units) was quantified at nine adherens junctions linking 10 consecutive cells using a fixed laser excitation time across all samples and all donors. A representative example of confocal image profiles at 48 hours is shown for control (Con, top left) and smoke-exposed (Smk, top right) cells, along with the calculated signal strength per junction (bottom left). Immunosignal intensity of E-cadherin in smoke-treated cells was normalized to untreated controls (designated as onefold) and reported as mean \pm SD fold changes (bottom right). ${ }^{*} P<$ $0.05, n=3$ donors. D: HBE cells in 3D culture were incubated for 48 hours with increasing concentrations of smoke-conditioned medium: $0 \%$ (Con), $25 \%$ and $50 \%$ Smk. E-cadherin immunoprecipitates were immunoblotted with anti- $\beta$-catenin antibody. Blots were stripped and re-probed with E-cadherin antibody. $\beta$-ctn, $\beta$-catenin; Con, control; E-Cad, E-Cadherin; IB, immunoblotting; IP, immunoprecipitation; Smk: smoke-conditioned medium. 
A
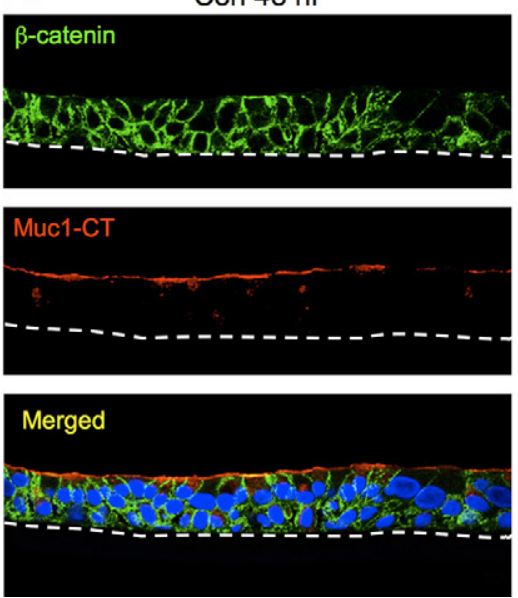

Smk $48 \mathrm{hr}$
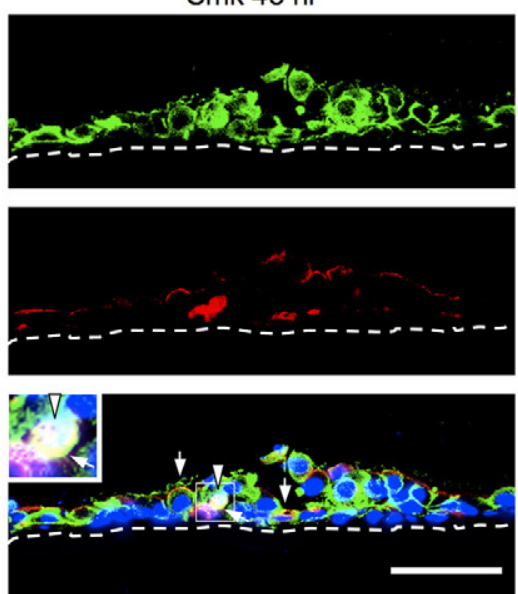

B

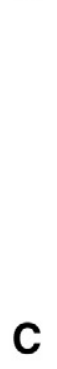

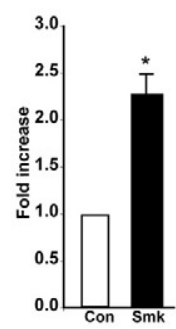

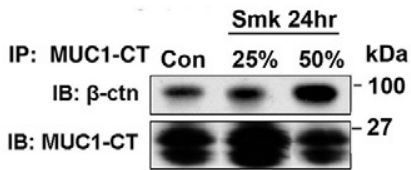

Figure 3. CS causes the intracellular formation and nuclear accumulation of MUC1-cytoplasmic tail/ $\beta$-catenin protein complexes. A: Immunolocalization of MUC1-CT and $\beta$-catenin in pseudostratified HBE cells following 48 hours of smoke exposure. By 48 hours of smoke exposure, MUC1-CT was redistributed from the apical plasma membrane to the cytoplasm in company with the loss of intercellular $\beta$-catenin. Colocalization of MUC1-CT (red) and $\beta$-catenin (green) in the cytoplasm generates a yellow signal (arrow), while colocalization of MUC1-CT (red) and $\beta$-catenin (green) in the cell nucleus (blue, as counterstained by DAPI) generates a white signal (arrowhead) in laser scanning confocal images. Scale bar $=50 \mu \mathrm{m}$. Dotted line delineates membrane surface of the $3 \mathrm{D}$ culture insert. B: MUC1-CT immunoprecipitates from nuclear cell lysates of three donors were probed with anti- $\beta$-catenin antibody following a 48 -hour exposure to $50 \%$ Smk-conditioned or control medium. Densitometric analysis of $\beta$-catenin immunoprecipitated by MUC1-CT in 50\% Smk-treated HBE cells was normalized to untreated control (designated as onefold) and are reported as mean \pm SD fold changes. $\left({ }^{*} P<0.05, n=3\right.$ donors). C: HBE cells were incubated for 24 hours with $0 \%$ (Con), $25 \%$ and $50 \%$ Smk. MUC1-CT immunoprecipitates from total cell lysates were probed with anti- $\beta$-catenin antibody. Blots were probed with MUC1-CT antibody as a loading control. $\beta$-ctn, $\beta$-catenin; Con, control; IB, immunoblot; IP, immunoprecipitation; HBE cells, human bronchial epithelial cells; MUC1-CT: MUC1-cytoplasmic tail; Smk: smoke-conditioned medium.

\section{Effects of CS on AJ Dissolution and MUC1-CT/ $\beta$-Catenin Interaction Are EGFR-Dependent}

EGFR, a growth factor receptor normally restricted to the basolateral plasma membrane domain of the bronchial epithelium, often has ectopic expression in lung cancer cells. ${ }^{27}$ We studied whether or not EGFR was a target of CS in our three-dimensional HBE cell model, and if so, whether its ectopic expression was important for CSinduced loss of AJs and redistribution of MUC1-CT/ $\beta$ catenin complexes. Within 48-hour of CS exposure, cilia on the apical cell surface disappeared and apical-basal polarization was disrupted throughout the entire epithelium (Figure 4A). Interestingly, EGFR inhibitor, AG 1478 (5 $\mu \mathrm{mol} / L)$, largely prevented CS-induced disorganization of the airway tissue (Figure 4A). Moreover, EGFR, which is normally restricted to the basolateral cell membrane, translocated intracellularly to the perinuclear region on CS exposure, and this effect was largely abolished in cells treated with AG1478 (Figure 4B). Increased presence of phosphorylated EGFR (pEGFR) in MUC1-CT immunoprecipitates suggested a direct physical interaction between pEGFR and MUC1-CT in response to CS that occurred over the same time frame as EGFR dislocation to the cytoplasm (Figure 4C). Moreover, the interaction of pEGFR with MUC1-CT was accompanied by EGFR-dependent tyrosine phosphorylation of MUC1-CT (Figure 4D). In addition to blocking EGFR phosphorylation and reducing MUC1-CT phosphorylation (Figure 4, C and D, respectively), AG 1478 largely attenuated the redistribution of apical MUC1-CT and junctional $\beta$-catenin as well as the formation of intracellular MUC1-CT/ $\beta$-catenin complexes (Figure 4E). Preservation of cell polarity in the presence of AG1478 was further demonstrated through persistent expression of E-cadherin at the AJs (Figure 4F) and the maintenance of $\beta$-catenin binding to $E$-cadherin at the AJ (Figure 4G). Taken together, inhibition of EGFR phosphorylation preserved the polarized distribution of EGFR, MUC1, and $\beta$-catenin, as well as the presence of E-cadherin at AJs in CS-treated HBE cells. These data suggest that loss of AJs and cell polarity, subcellular re-distribution of EGFR, MUC1, and $\beta$-catenin, as well as MUC1-CT/ $\beta$-catenin complex formation in response to CS are initiated through an EGFR-dependent pathway.

\section{CS-Elicited Up-Regulation and Nuclear Recruitment of Wnt Nuclear Effector, Lef-1, Is EGFR-Dependent}

Given the accumulation of MUC1-CT/ $\beta$-catenin complexes in the nucleus of HBE cells in response to CS (Figure 4), we investigated whether or not CS affected the activity of $\beta$-catenin-mediated canonical Wnt signaling in pseudostratified HBE cells by assessing LEF- 1 transcriptional activity and the cyto-localization of Lef- 1 protein. Lef- 1 is the direct nuclear effector of $\beta$-catenin and functions as cotranscription factor through which $\beta$-catenin transactivates a wide array of Wnt downstream target genes. ${ }^{28}$ Using TaqMan PCR, we observed significant up-regulation of LEF-1 during exposure to $50 \%$ Smk for 48 hours, and this effect was abolished by AG1478. Intriguingly, blocking the Wnt receptor with Dkk-1 did not significantly attenuate CS-induced LEF-1 up-regulation (Figure 5, A and B). These data suggest that CS may primarily activate Wnt signaling through an EGFR-dependent and Wnt ligand-receptor-independent mechanism. With confocal imaging, we confirmed the nuclear local- 
A

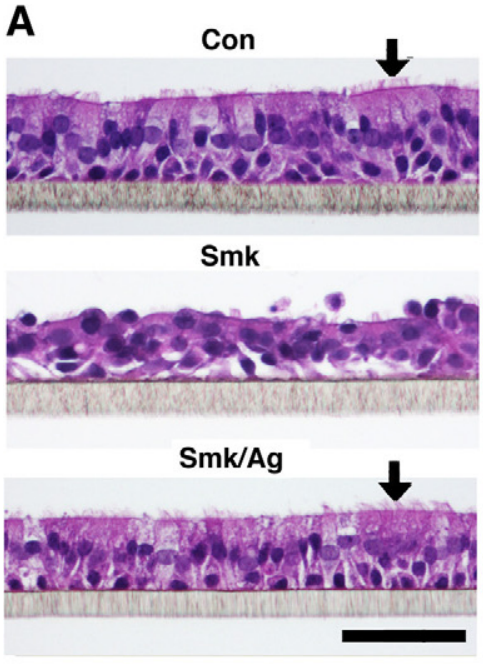

E
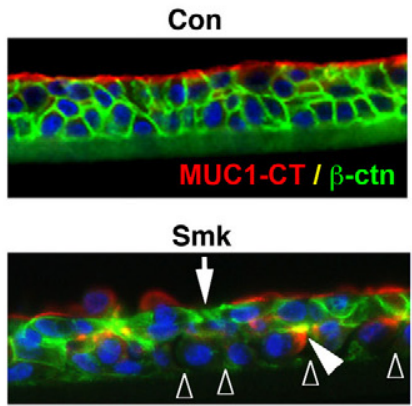

Smk/Ag

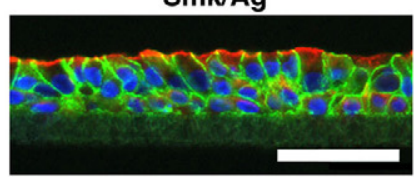

B

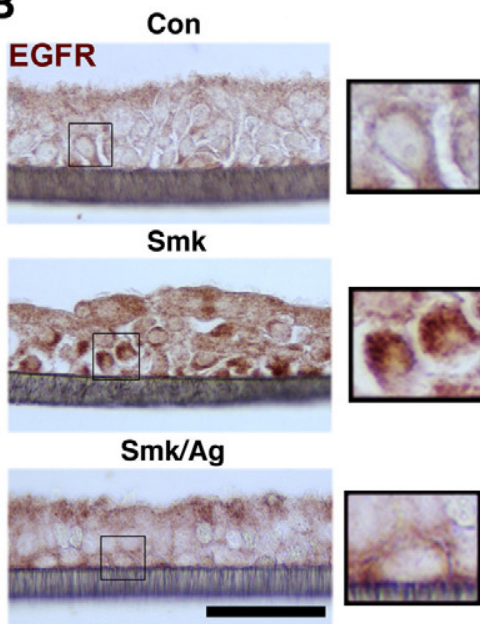

$\mathbf{F}$
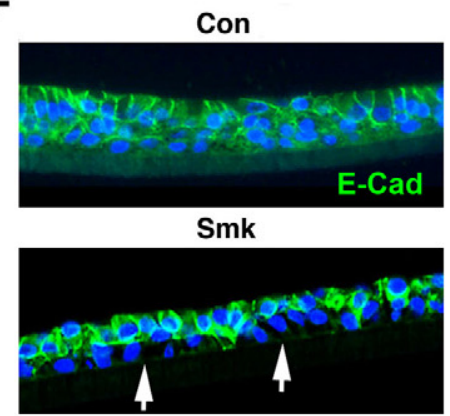

Smk/Ag

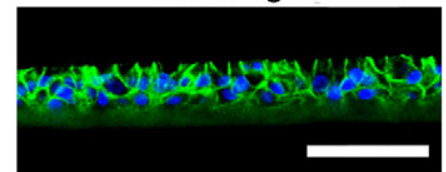

C

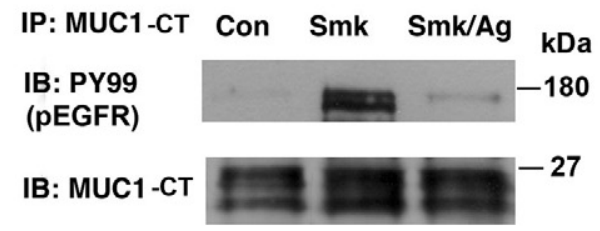

D

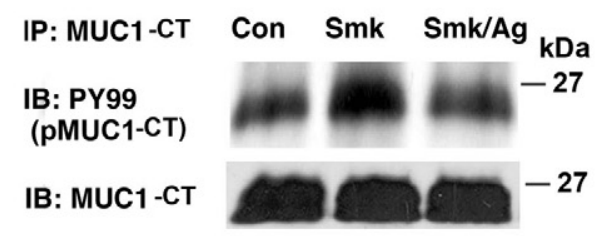

G

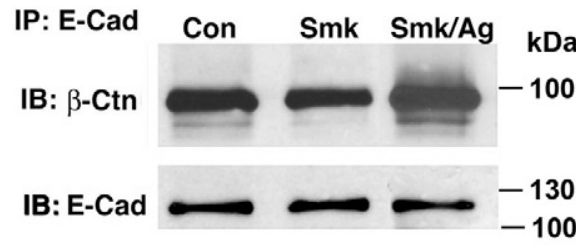

Figure 4. Effects of CS on structural change and cell polarity of airway epithelium are EGFR-dependent. A: H\&E staining of pseudostratified HBE cells exposed to $50 \%$ Smk for 48 hours in the presence ( $\mathrm{mmk} / \mathrm{Ag}$ ) and absence (Smk) of EGFR inhibitor, AG1478. Untreated cells were used as controls (Con). Black arrows indicate ciliated apical cells in normal polarized epithelium. Scale bars $=50 \mu \mathrm{m}$. B: Immunohistochemistry to localize EGFR in pseudostratified HBE cells exposed to $50 \%$ Smk for 48 hours with or without AG1478. EGFR at the cell membrane in control cells (upper inset) moves to perinuclear sites in response to smoke (middle inset). Smoke exposure in the presence of AG1478 prevents perinuclear accumulation of EGFR and leaves it undisturbed at the cell membrane (lower inset). To better demonstrate perinuclear distribution of EGFR, cell nuclei were not counterstained. Scale bars $=50 \mu \mathrm{m}$. C and D: Pseudostratified HBE cells were incubated with $50 \%$ Smk for four hours in the presence (Smk/Ag) or absence (Smk) of EGFR inhibitor, AG1478. Untreated cells were used as controls (Con). Lysates immunoprecipitated by MUC1-CT antibody were immunoblotted with anti-phospho-tyrosine antibody (PY99). Blots were then stripped and reprobed with anti-MUC1-CT for loading control. Tyrosine phosphorylation of (C) EGFR (179 kDa) and (D) MUC1-CT ( 16-26 kDa) were determined on the basis of molecular weight and verified by protein ladder. E and F: Immunofluorescent staining of (E) MUC1-CT/ $\beta$-catenin and (F) E-cadherin was performed in stratified HBE cells exposed to $50 \%$ smoke (Smk) with or without AG1478 (Smk/Ag) for 48 hours. Cell nuclei were counterstained with DAPI (blue). Scale bars $=50 \mu \mathrm{m}$. Of note, the loss of E-cadherin at the intercellular junctions (arrow in F) coincided with the loss of apical MUC1-CT (white arrow in E) and junctional $\beta$-catenin (open arrowheads in E). Intracellular translocation of MUC1-CT/ $\beta$-catenin complexes noted in Smk (white arrowhead in E) was not seen in Smk/Ag. G: Cell lysates from the same preparation of $50 \%$ Smk-treated HBE cells as in $\mathbf{C}$ and $\mathbf{D}$ were further immunoprecipitated with anti-E-cadherin antibody and immunoblotted with anti- $\beta$-catenin. E-cadherin was probed as the loading control. All data are representative of at least three different donors. $\beta$-ctn, $\beta$-catenin; Con, control; E-Cad, E-cadherin; IB, immunoblotting; IP, immunoprecipitation; PY99, anti-phospho-tyrosine antibody; Smk: 50\% smoke-conditioned medium; Smk/Ag: 50\% smoke-conditioned medium with $1 \mu \mathrm{mol} / \mathrm{L}$ AG1478.

ization of Lef-1 transcription factor in response to CS. Lef-1 accumulated in a punctate pattern in the cell nucleus of CS-treated HBE cells, indicating activation of canonical Wnt signaling (Figure 5C). ${ }^{29}$ Nuclear localization of Lef-1 was inhibited by AG1478, indicating a link between upstream EGFR signaling and downstream Wnt signaling in CS airway pathology. Collectively, these data supported our hypothesis that loss of cell polarity, nuclear accumulation of MUC1-CT/ $\beta$-catenin complexes, increased Wnt nuclear effector Lef-1, and aberrant activation of canonical Wnt signaling in response to CS occured via an EGFR-dependent mechanism.

\section{Discussion}

Although the correlation between CS and lung cancer is well-established and key genes have been identified through multistage molecular epidemiology studies, ${ }^{30}$ the fundamental link between CS-induced structural changes of the airway epithelium and the onset of tumorigenesis remains poorly defined. Using a $3 \mathrm{D}$ in vitro model of the airway epithelium, we demonstrated an interdependent relationship between CS-induced disruption of epithelial cell polarity and aberrant activation of canonical Wnt signaling that could contribute to tumorigenesis. 
A

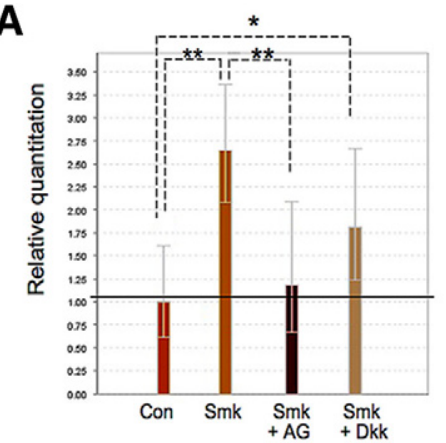

C

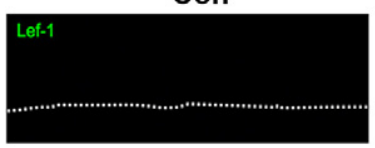

P Merged

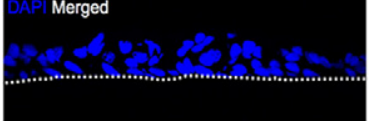

B

\begin{tabular}{|c|c|c|c|c|}
\hline \multicolumn{5}{|c|}{ TaqMan Gene Expression Assay } \\
\hline & Con & Smk & $\begin{array}{l}\text { Smk + } \\
\text { AG1478 }\end{array}$ & $\begin{array}{l}\text { Smk + } \\
\text { Dkk1 }\end{array}$ \\
\hline$\Delta \mathrm{C}_{\mathrm{T}}$ Mean & 18.27 & 16.87 & 18.03 & 17.41 \\
\hline$\Delta \mathrm{C}_{\mathrm{T}} \mathrm{SD}$ & 0.64 & 0.33 & 0.80 & 0.53 \\
\hline$R Q$ & 1 & 2.65 & 1.18 & 1.81 \\
\hline$p$ value & ref & 0.007 & 0.326 & 0.043 \\
\hline$p$ valuu & 0.007 & ref & 0.003 & 0.110 \\
\hline
\end{tabular}

Smk
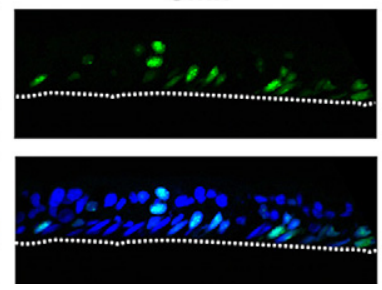

Smk/Ag
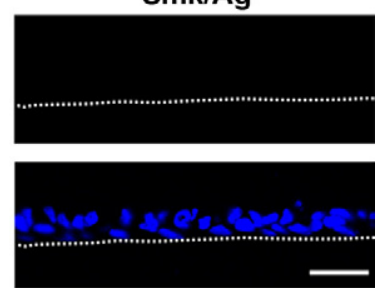

Figure 5. Up-regulation of LEF1 transcription and nuclear localization of Lef-1 protein in CS-treated HBE cells are EGFR-dependent. A: Real-time RTPCR using TaqMan gene expression assay to examine transcriptional activity of Wnt downstream effector Lef-1 in pseudostratified HBE cells following 48-hour exposure to smoke (Smk) in the presence or absence of EGFR inhibitor, AG1478 $(\mathrm{Smk}+\mathrm{AG})$ or Wnt antagonist, Dkk-1 (Smk + Dkk). Data are presented as relative fold increases (Mean $\pm \mathrm{SD}$ ), compared with control (Con) defined as onefold. ${ }^{*} P<0.05,{ }^{* *} P<0.01$. B: $\Delta$ CT value (mean $\pm \mathrm{SD})$, relative quantitation $(\mathrm{RQ})$, and $P$ value (analysis of variance) are reported. AG1478, $1 \mu \mathrm{mol} / \mathrm{L} ;$ Dkk-1, $100 \mu \mathrm{mol} / \mathrm{L}$. C: Nuclear localization of Lef-1 in HBE cells exposed to 50\% Smk for 48 hours. Cell nuclei were counterstained with DAPI (blue). Scale bar $=50 \mu \mathrm{m}$. Dotted line delineates membrane surface of the culture insert. Data are representative of three different donors.
Cell polarity is critical for proper tissue function, including the control of cell proliferation, survival and differentiation. Specific localization of different membrane proteins in different cell surface domains is a feature common to many types of polarized cells, including epithelia, neurons, and immune cells. ${ }^{31}$ In airway epithelial cells, membrane proteins are segregated into functionally and structurally different apical and basolateral membrane domains. The establishment and maintenance of cell polarity depends on the formation of junctional complexes that include both AJs and TJs. E-cad- herin, together with $\beta$-catenin, is a key component of the AJ. ${ }^{32}$ During CS exposure, we observed loss of cell polarity (Figure 1B) and a progressive decrease in E-cadherin (Figure 2). Initially, E-cadherin decreased in the basal cell layer where intercellular spaces were increasing (Figure 4F, Smk), indicating that the dissociation of E-cadherin-mediated AJs is related to the loss of epithelial integrity and polarity. In the pseudostratified, bronchial epithelium, E-cadherin serves as a marker for epithelial differentiation, with higher expression in the superficial ciliated layer and lower expression in the un-
A

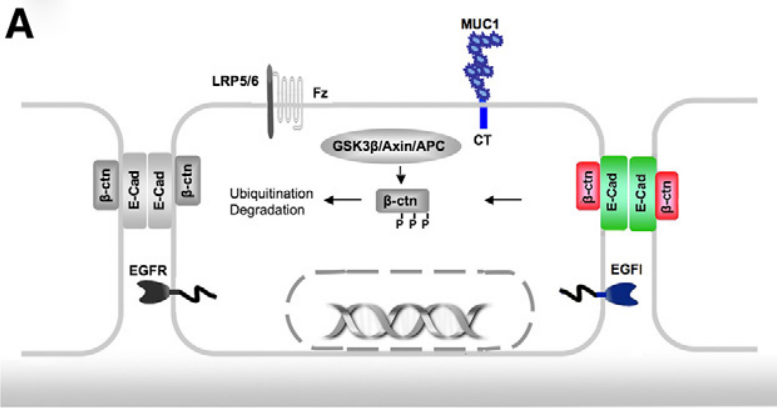

B

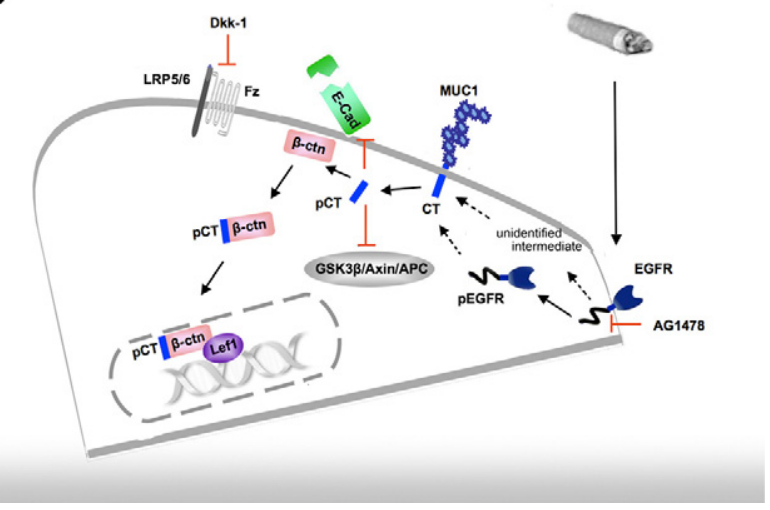

Figure 6. Hypothetical model for EGFR-dependent loss of cell polarity and activation of Wnt signaling in CS-exposed human airway epithelium. A: The cell membrane of polarized airway epithelial cells is separated into apical and basolateral domains by intercellular junctional complexes located at the apicolateral cell membrane. MUC1 on the apical membrane, EGFR on the basolateral membrane, and $\beta$-catenin in complex with E-cadherin at the AJs (as part of junctional complex) form landmarks that delineate a polarized epithelial surface. Under normal physiological conditions, Wnt signaling occurs via the cell surface receptor, Frizzled (Fz) is off, and intracellular $\beta$-catenin released from the AJs is targeted by the GSK3 $\beta /$ Axin/APC complex for ubiquitination and degradation. B: On CS exposure, basolateral membrane-restricted EGFR is phosphorylated and translocated intracellularly. The cytoplasmic tail (CT) of transmembrane-MUC1 physically interacts with intracellularly translocated pEGFR and is phosphorylated either directly by pEGFR or through unidentified intermediates. Via an EGFR-dependent mechanism, MUC1-CT is cleaved from its extracellular mucin domain and released intracellularly where it forms a complex with $\beta$-catenin. MUC1-CT, E-cadherin, and adenomatous polyposis coli (APC) share a common binding motif structure for $\beta$-catenin; thus, MUC1-CT might potentially contribute to loss of cell-cell adhesion through competitive binding of $\beta$-catenin away from its complex with E-cadherin at the AJs as well as stabilize $\beta$-catenin by preventing APC complex-mediated $\beta$-catenin degradation. In complex with MUC1-CT, $\beta$-catenin accumulates in the cell nucleus and subsequently leads to the transactivation of Wnt downstream nuclear target genes through the binding of its cotranscriptional factor, Lef-1 on DNA. The aberrant activation of canonical $\mathrm{Wnt} / \beta$-catenin signaling in response to smoke is distinct from the "conventional" counterpart in that the smoke-elicited one is primarily EGFR-dependent (inhibited by AG1478) and the conventional counterpart is typically Wnt receptordependent (inhibited by Dkk-1). Structural molecules related to cell polarity and signaling molecules related to the $\mathrm{Wnt} / \beta$-catenin pathway activation in response to CS are highlighted in colors. Dotted lines indicate hypothetical pathways that await further study. 
differentiated basal layer. Interestingly, when HBE cells were immersed in CS-conditioned medium, we observed a time-dependent decrease in E-cadherin that was initiated in the basal layer (Figure 4) and eventually progressed throughout the pseudostratified epithelium. In company with loss of E-cadherin, basolateral EGFR was internalized and redistributed to the perinuclear area (Figure 4B). Both ligand-dependent and ligand-independent mechanisms have been reported in CS-induced EGFR phosphorylation, cytosolic localization, and signaling in HBE monolayer culture. ${ }^{21,22}$ Although the mechanism whereby EGFR undergoes intracellular translocation in the current 3D model awaits further study, our data demonstrate that EGFR activation by tyrosine phosphorylation is essential to the detrimental effects of CS on epithelial cell polarity (Figure 4). Blocking EGFR with its tyrosine kinase inhibitor, AG 1478, maintained cell polarity in response to CS by preventing dissociation of AJs and intracellular dislocation of apical mucin, MUC1 (Figure $4, A, B, E$, and F). AJs and TJs are the major junctional complexes that serve to establish and maintain cell polarity. ${ }^{33}$ Intriguingly, a similar EGFR-dependent mechanism was also found to mediate the damaging effects of CS on TJS, and this event was completely abolished in the presence of AG1478. ${ }^{34}$ These results demonstrate EGFR's essential role in mediating the damaging effects of CS on cell-cell adhesion. Whether EGFR acts alone or in combination with other cell surface receptors will require further exploration.

An important pathophysiological implication of CS-induced AJ disassembly is the release of $\beta$-catenin from its physical binding with E-cadherin at the apicolateral membrane, thereby promoting its potential role as an intracellular activator of Wnt signaling. On release from the AJs, our data showed intracellular translocation and nuclear accumulation of $\beta$-catenin, as well as increased immunolocalization of Lef- 1 in the nucleus. Taken together, these results provide evidence that key events essential for activating transcription of Wnt downstream target genes are occurring in response to CS exposure (Figures 3A and $5 \mathrm{C}$ ). Accordingly, in preliminary studies using SYBR Green-based quantitative PCR, we found that CS upregulated two established nuclear targets of $\mathrm{Wnt} / \beta$-catenin/Lef-1 signaling pathway, WISP1 (WNT1 inducible signaling pathway protein1, 5.86-fold-increase) and FGF4 (fibroblast growth factor 4, 19.45-fold-increase) (unpublished data). ${ }^{35,36}$ Although the activation of canonical Wnt signaling has been reported in previous publications using HBE cell monolayers, ${ }^{10,23}$ to the best of our knowledge this study is the first to demonstrate CS-induced activation of Wnt signaling in a fully differentiated pseudostratified model of the human airway epithelium that compromises three heterogeneous cell populations (ciliated columnar cells, goblet cells, and undifferentiated basal cells). Moreover, we established a pathological link between upstream EGFR and downstream canonical Wnt signaling in response to CS exposure. This novel EGFRdependent pathway adds more complexity to Wnt signaling-mediated airway tissue remodeling in CS airway pathology. Interestingly, CS has also been reported to activate canonical Wnt signaling through a Wnt receptor-dependent mechanism in the alveolar epithelium of the distal airway via the inhibition of glycogen synthase kinase $3 \beta($ GSK $3 \beta){ }^{10}$ The effects of CS on normal nontransformed airway cells appear to differ from those that are malignantly-transformed. For example, Hussain et al showed that CS directly engaged polycomb machinery to activate canonical Wnt signaling in the maintenance of cancer stem cells, ${ }^{37}$ while Marsit et al reported CS-induced Wnt signaling through epigenetic silencing of the extracellular Wnt antagonist, soluble frizzled receptor protein (SFRP). ${ }^{38}$ The complexity of mechanisms regulating canonical Wnt signaling in response to CS might reflect a stage-specific control of Wnt signaling activation during the multistep process of malignant transformation. The significance the EGFR-dependent effects of smoke on airway epithelial polarity and the mechanism whereby it contributes to preneoplastic "field cancerization" warrants further study.

In addition to the effects on CS on mobilizing EGFR and $\beta$-catenin, we observed loss of membrane-bound mucin, MUC1, at the apical epithelial surface, along with intracellular translocation of its cytoplasmic tail (Figure 3A). This mucin change occurs in company with disappearance of cilia (Figure 1B), disassembly of AJs (Figure 2), and intracellular complex formation between MUC1-CT and $\beta$-catenin (Figure 3C). Although intracellular translocation of MUC1-CT is frequently observed in various tumor cells, ${ }^{39}$ the biological significance of its intracellular localization in complex with $\beta$-catenin in a nonmalignant model of the airway epithelium remains unclear. Accumulating evidence suggests that the cytoplasmic tail of MUC1 interacts with various growth factor receptors and $\beta$-catenin. ${ }^{12}$ Interestingly, MUC1-CT, E-cadherin and APC share a common binding motif (SXXXXXSSL) for $\beta$-catenin. ${ }^{40}$ In breast cancer, intracellularly overexpressed MUC1 glycoprotein interferes with E-cadherin-mediated cell-cell adhesion and APC-mediated degradation of $\beta$-catenin by forming MUC1$\mathrm{CT} / \beta$-catenin complexes. ${ }^{41,42}$ Further study to determine whether CS induces intracellularly dislodged MUC1-CT to compete with E-cadherin for $\beta$-catenin binding at the AJs and/or serves as a docking site to shield $\beta$-catenin from APC complex ubiquitination will undoubtedly uncover the biological significance of CS-induced MUC1$\mathrm{CT} / \beta$-catenin complex formation in the polarized airway. A putative mechanism is illustrated in Figure $6, \mathrm{~A}$ and $\mathrm{B}$.

In our 3D model of the bronchial epithelium that closely mimics the in vivo human airway, EGFR appears to serve as a gateway through which CS disrupts cell polarity and aberrantly activates canonical Wnt signaling. In the midst of complex signaling networks induced by CS, a pathological link between cell polarity and tumorigenic Wnt-signaling pathway might offer a new perspective in understanding the early premalignant stages of tissue architecture remodeling in multistep lung carcinogenesis.

\section{Acknowledgments}

We thank Dr. Kent Pinkerton (University of California Davis) for providing the smoke conditioned medium, 
Dr. Sandra Gendler (Mayo Clinic, Scottsdale) for proving anti-MUC1-CT2, and the technical assistance of Sebastian Peck with confocal imaging (Biological Imaging Development Center, University of California, San Francisco).

\section{References}

1. Guindon GE, de Beyer J, Galbraith S: Framework convention on tobacco control: progress and implications for health and the environment. Environ Health Perspect 2003, 111:A262-A263

2. Hamadeh RR, McPherson K, Doll R: Tobacco consumption and chemical analysis of cigarettes in Bahrain. Int $J$ Addict 1994, 29:325-337

3. Proctor RN: Tobacco and the global lung cancer epidemic. Nat Rev Cancer 2001, 1:82-86

4. Gritz ER, Dresler C, Sarna L: Smoking, the missing drug interaction in clinical trials: ignoring the obvious. Cancer Epidemiol Biomarkers Prev 2005, 14:2287-2293

5. Spina D: Epithelium smooth muscle regulation and interactions. Am J Respir Crit Care Med 1998, 158:S141-S145

6. Knight DA, Holgate ST: The airway epithelium: structural and functional properties in health and disease. Respirology 2003, 8:432-446

7. Hattrup CL, Gendler SJ: Structure and function of the cell surface (tethered) mucins. Annu Rev Physiol 2008, 70:431-457

8. Pohl C, Hermanns MI, Uboldi C, Bock M, Fuchs S, Dei-Anang J, Mayer E, Kehe K, Kummer W, Kirkpatrick CJ: Barrier functions and paracellular integrity in human cell culture models of the proximal respiratory unit. Eur J Pharm Biopharm 2009, 72:339-349

9. Xu W, Kimelman D: Mechanistic insights from structural studies of beta-catenin and its binding partners. J Cell Sci 2007, 120:3337-3344

10. Tian D, Zhu M, Li J, Ma Y, Wu R: Cigarette smoke extract induces activation of beta-catenin/TCF signaling through inhibiting GSK3beta in human alveolar epithelial cell line. Toxicol Lett 2009, 187:58-62

11. Mazieres J, He B, You L, Xu Z, Jablons DM: Wnt signaling in lung cancer. Cancer Lett 2005, 222:1-10

12. Carson DD: The cytoplasmic tail of MUC1: a very busy place. Sci Signal 2008, 1:pe35

13. Hollingsworth MA, Swanson BJ: Mucins in cancer: protection and control of the cell surface. Nat Rev Cancer 2004, 4:45-60

14. Wistuba II, Gazdar AF: Lung cancer preneoplasia. Annu Rev Pathol 2006, 1:331-348

15. Slaughter DP, Southwick HW, Smejkal W: Field cancerization in oral stratified squamous epithelium; clinical implications of multicentric origin. Cancer 1953, 6:963-968

16. Steiling K, Ryan J, Brody JS, Spira A: The field of tissue injury in the lung and airway. Cancer Prev Res (Phila Pa) 2008, 1:396-403

17. Lee JJ, Liu D, Lee JS, Kurie JM, Khuri FR, Ibarguen H, Morice RC, Walsh G, Ro JY, Broxson A, Hong WK, Hittelman WN: Long-term impact of smoking on lung epithelial proliferation in current and former smokers. J Natl Cancer Inst 2001, 93:1081-1088

18. Wistuba II, Mao L, Gazdar AF: Smoking molecular damage in bronchial epithelium. Oncogene 2002, 21:7298-7306

19. Martinez-Garcia E, Irigoyen M, Anso E, Martinez-Irujo JJ, Rouzaut A Recurrent exposure to nicotine differentiates human bronchial epithelial cells via epidermal growth factor receptor activation. Toxicol Appl Pharmacol 2008, 228:334-342

20. Griffin S, Carroll TP, Greene CM, O'Neill SJ, Taggart CC, McElvaney NG: Effect of pro-inflammatory stimuli on mucin expression and inhibition by secretory leucoprotease inhibitor. Cell Microbiol 2007, 9:670-679

21. Khan EM, Lanir R, Danielson AR, Goldkorn T: Epidermal growth factor receptor exposed to cigarette smoke is aberrantly activated and undergoes perinuclear trafficking. FASEB J 2008, 22:910-917

22. Lemjabbar H, Li D, Gallup M, Sidhu S, Drori E, Basbaum C: Tobacco smoke-induced lung cell proliferation mediated by tumor necrosis factor alpha-converting enzyme and amphiregulin. J Biol Chem 2003, 278:26202-26207

23. Lemjabbar-Alaoui H, Dasari V, Sidhu SS, Mengistab A, Finkbeiner W, Gallup M, Basbaum C: Wnt and Hedgehog are critical mediators of cigarette smoke-induced lung cancer. PLoS One 2006, 1:e93

24. Yamaya M, Finkbeiner WE, Chun SY, Widdicombe JH: Differentiated structure and function of cultures from human tracheal epithelium. Am J Physiol 1992, 262:L713-L724

25. Fulcher ML, Gabriel S, Burns KA, Yankaskas JR, Randell SH: Welldifferentiated human airway epithelial cell cultures. Methods Mol Med 2005, 107:183-206

26. Ji CM, Plopper CG, Witschi HP, Pinkerton KE: Exposure to sidestream cigarette smoke alters bronchiolar epithelial cell differentiation in the postnatal rat lung. Am J Respir Cell Mol Biol 1994, 11:312-320

27. Wei EX, Anga AA, Martin SS, Jackson JG, Nordberg ML, Herrera GA Turbat-Herrera EA: EGFR expression as an ancillary tool for diagnosing lung cancer in cytology specimens. Mod Pathol 2007, 20:905-913

28. Behrens J, von Kries JP, Kuhl M, Bruhn L, Wedlich D, Grosschedl R, Birchmeier W: Functional interaction of beta-catenin with the transcription factor LEF-1. Nature 1996, 382:638-642

29. Kato N, Shimmura S, Kawakita T, Miyashita H, Ogawa Y, Yoshida S, Higa K, Okano H, Tsubota K: Beta-catenin activation and epithelialmesenchymal transition in the pathogenesis of pterygium. Invest Ophthalmol Vis Sci 2007, 48:1511-1517

30. Minna JD, Roth JA, Gazdar AF: Focus on lung cancer. Cancer Cell 2002, 1:49-52

31. Bryant DM, Mostov KE: From cells to organs: building polarized tissue. Nat Rev Mol Cell Biol 2008, 9:887-901

32. Knust E, Bossinger O: Composition and formation of intercellular junctions in epithelial cells. Science 2002, 298:1955-1959

33. Wodarz A, Nathke I: Cell polarity in development and cancer. Nat Cell Biol 2007, 9:1016-1024

34. Petecchia L, Sabatini F, Varesio L, Camoirano A, Usai C, Pezzolo A, Rossi GA: Bronchial airway epithelial cell damage following exposure to cigarette smoke includes disassembly of tight junction components mediated by the extracellular signal-regulated kinase 1/2 pathway. Chest 2009, 135:1502-1512

35. Shackleford GM, MacArthur CA, Kwan HC, Varmus HE: Mouse mammary tumor virus infection accelerates mammary carcinogenesis in Wnt-1 transgenic mice by insertional activation of int-2/Fgf-3 and hst/Fgf-4. Proc Natl Acad Sci USA 1993, 90:740-744

36. Tian C, Zhou ZG, Meng WJ, Sun XF, Yu YY, Li L, Luo HZ, Yang L, Zhou B, Gu J: Overexpression of connective tissue growth factor WISP-1 in Chinese primary rectal cancer patients. World J Gastroenterol 2007, 13:3878-3882

37. Hussain M, Rao M, Humphries AE, Hong JA, Liu F, Yang M, Caragacianu D, Schrump DS: Tobacco smoke induces polycomb-mediated repression of Dickkopf-1 in lung cancer cells. Cancer Res 2009, 69:3570-3578

38. Marsit CJ, McClean MD, Furniss CS, Kelsey KT: Epigenetic inactivation of the SFRP genes is associated with drinking, smoking and HPV in head and neck squamous cell carcinoma. Int J Cancer 2006, 119:1761-1766

39. Guddo F, Giatromanolaki A, Koukourakis MI, Reina C, Vignola AM, Chlouverakis G, Hilkens J, Gatter KC, Harris AL, Bonsignore G: MUC1 (episialin) expression in non-small cell lung cancer is independent of EGFR and C-erbB-2 expression and correlates with poor survival in node positive patients. J Clin Pathol 1998, 51:667-671

40. Yamamoto M, Bharti A, Li Y, Kufe D: Interaction of the DF3/MUC1 breast carcinoma-associated antigen and beta-catenin in cell adhesion. J Biol Chem 1997, 272:12492-12494

41. Li Y, Bharti A, Chen D, Gong J, Kufe D: Interaction of glycogen synthase kinase 3beta with the DF3/MUC1 carcinoma-associated antigen and beta-catenin. Mol Cell Biol 1998, 18:7216-7224

42. Hattrup CL, Fernandez-Rodriguez J, Schroeder JA, Hansson GC Gendler SJ: MUC1 can interact with adenomatous polyposis coli in breast cancer. Biochem Biophys Res Commun 2004, 316:364-369 